\title{
Tratamento farmacológico da Paralisia Facial Periférica Idiopática: qual a evidência?
}

Ana Isabel Silva,* Tiago Magalhães**

\section{RESUMO}

Objetivo: Rever o tratamento farmacológico mais eficaz, entre corticoides e antivíricos, na melhoria clínica da Paralisia Facial Periférica Idiopática.

Fontes de dados: Pubmed, sítios de medicina baseada na evidência, Índex de Revistas Médicas Portuguesas e referências bibliográficas dos artigos selecionados.

Métodos de Revisão: Foi realizada uma pesquisa de normas de orientação clínica, revisões sistemáticas, meta-análises (MA) e ensaios clínicos aleatorizados e controlados, publicados entre 01/2005 e 04/2012, em inglês e português, utilizando os termos MeSH: Glucocorticoids; Antiviral Agents; Bell Palsy; Facial Paralysis. Para avaliação dos níveis de evidência (NE) e atribuição de forças de recomendação foi utilizada a escala Strength of Recommendation Taxonomy (SORT) da American Family Physician.

Resultados: Foram encontrados 181 artigos, dos quais foram selecionados sete MA. Três MA suportam de forma consistente e significativa o benefício clínico do uso de corticoide versus placebo (NE 1). O uso de antivíricos não foi melhor que o placebo em dois estudos (NE 2). A combinação de corticoide e antivírico foi semelhante ao corticoide isolado em dois estudos e em quatro estudos foi reportado um benefício da combinação relativamente ao uso isolado de corticoide, sem significado estatístico. Este benefício da combinação de corticoide e antivírico foi potencialmente mais relevante em casos de paralisia severa (NE 2).

Conclusões: A corticoterapia continua a ser o tratamento com a melhor evidência clínica (Recomendação A). Não existe evidência de benefício dos antivíricos, isoladamente ou em associação com corticoides, no tratamento da Paralisia de Bell (Recomendação B). Em doentes com paralisia severa poderá, potencialmente, existir um pequeno benefício na adição de antivíricos à terapêutica com corticoides (Recomendação B). São de realçar as diversas limitações metodológicas, assim como a heterogeneidade dos estudos.

Palavras-chave: Paralisia Facial Periférica Idiopática; Paralisia de Bell; Corticosteroides; Antivíricos.

\section{INTRODUÇÃO}

1 Paralisia de Bell ou Paralisia Facial Periférica Idiopática (PFPI) consiste numa paralisia ou parésia aguda do nervo facial, geralmente unilateral, de causa desconhecida, sendo caracterizada por inflamação e edema deste nervo ao longo do seu trajeto pelo ouvido interno, o que pode resultar numa compressão e desmielinização axonal, com diminuição da irrigação sanguínea. ${ }^{1,2}$

A sua incidência anual situa-se entre os 20 e os 30 por

*A.I.S.: Unidade de Saúde Familiar Nova Salus - ACeS Grande Porto VII - Gaia; Interna de Medicina Geral e Familiar

**T.M.: Unidade de Cuidados de Saúde Personalizados Oliveira do Douro - ACeS Grande Porto VII - Gaia; Interno de Medicina Geral e Familiar
100.000 , com um pico por volta dos 20 a 40 anos, atingindo ambos os géneros em proporções similares. ${ }^{1,3}$ Existe uma maior prevalência nas grávidas e em doentes com diabetes mellitus, síndrome gripal ou outra patologia respiratória superior. ${ }^{1,4}$

No que concerne às etiologias suspeitas, consistem essencialmente na infeção vírica (com o vírus herpes simples 1 tendo uma probabilidade de ser o causador em $67 \%$ e havendo $33 \%$ de probabilidade de ocorrer uma reativação do vírus varicela-zoster), isquemia vascular, alterações inflamatórias autoimunes ou fatores hereditários. ${ }^{5}$

Sendo o diagnóstico essencialmente clínico, a recolha de uma boa história clínica e um exame físico direcionado são imprescindíveis. 
A PFPI apresenta-se clinicamente por incapacidade no encerramento do olho afetado na totalidade, desvio inferior/incapacidade para levantar a sobrancelha, diminuição do lacrimejo e/ou secreções salivares, parésia da boca do lado afetado, com desvio e apagamento do sulco, dificuldade na alimentação (sólidos e/ou líquidos - que muitas vezes referem escorregar pelo canto da boca), otalgia ao redor ou atrás da orelha (estendendo-se muitas vezes à região occipital/cervical), hiperacúsia com tolerância diminuída para níveis normais de ruído, perturbação do paladar nos $2 / 3$ anteriores da língua e stress psicológico marcado com eventual restrição das atividades sociais. ${ }^{1,6}$ Por sua vez, no exame físico será importante a distinção entre paralisia facial periférica ou central. Na central, o movimento da região frontal é normal bilateralmente, ou seja, esta zona é capaz de se «enrugar»; já na periférica, um dos lados tem paralisia ou parésia em toda a face, incluindo a região frontal do lado afetado. ${ }^{7}$ Uma otoscopia sem alterações significativas será importante para o diagnóstico de PFPI.

A escala de House-Brackmann é uma das mais utilizadas na classificação das PFPI, ${ }^{3}$ sendo uma das referidas por todas as meta-análises selecionadas para esta revisão, além de permitir comparações com outras escalas e ter sido adotada como padrão pela Facial Nerve Disorders Committee da American Academy of Otolaryngology - Head and Neck Surgery. ${ }^{4,5}$

Nesta escala, os graus de disfunção são definidos como: 1 -normal; 2 -ligeira (fraqueza apenas numa inspeção cuidadosa); 3 - moderada (óbvia, mas não desfigurante); 4 - moderadamente severa (fraqueza óbvia e/ou assimetria desfigurante); 5 - movimentos faciais praticamente não percetíveis; 6 - ausência de movimentos. $^{4}$

Os doentes não tratados podem ter uma recuperação completa em cerca de $70 \%$ dos casos, mas, em até $30 \%$, podem apresentar apenas uma recuperação incompleta do controlo muscular facial, podendo adquirir desfiguração facial, dor facial e distúrbios psicológi$\cos ^{1,6}$ Também foi demonstrado que a severidade inicial influencia o prognóstico. ${ }^{3}$

Deste modo, o objetivo do tratamento da PFPI consiste na prevenção das sequelas, sendo que as hipóteses atuais de tratamento farmacológico englobam os corticoides, antivíricos, ou a combinação de ambos.
Esta revisão tem como objetivo rever qual o tratamento farmacológico mais eficaz, entre corticoides e/ou antivíricos, na melhoria clínica da Paralisia Facial Periférica Idiopática.

\section{MÉTODOS}

Foi efetuada uma pesquisa nas bases de dados da Cochrane Library, Pubmed, National Guideline Clearinghouse, Dare, Tripdatabase, Bandolier, UpToDate e Índex de Revistas Médicas Portuguesas. Foi feita a pesquisa de normas de orientação clínica, meta-análises, artigos de revisão e ensaios clínicos aleatorizados e controlados, publicadas entre janeiro de 2005 e abril de 2012, em português e inglês, utilizando os termos Mesh: Glucocorticoids; Antiviral Agents; Bell Palsye Facial Paralysis. Estes termos foram utilizados utilizando operados booleanos, através da combinação (Glucocorticoids AND/OR Antiviral Agents) AND (Bell Palsy AND/OR Facial Paralysis), de modo a maximizar a possibilidade de encontrar todos os artigos potencialmente relevantes.

Foram incluídos artigos que respeitavam os critérios: população alvo constituída por indivíduos com o diagnóstico de PFPI, comparação do uso de corticoide e/ou antivírico versus placebo ou comparação da associação de corticoide e antivírico versus corticoide ou versus antivírico, sem restrições relativas ao tempo de evolução/seguimento posteriores ao tratamento testado.

Foram excluídos os artigos que incluíssem: doentes imunodeprimidos, grávidas ou lactentes, causas conhecidas de paralisia facial periférica (como a etiologia vascular, traumática, neoplásica), paralisia facial central ou artigos repetidos ou já alvo de avaliação em meta-análises/revisões sistemáticas.

Para avaliar a qualidade dos estudos e a força de recomendação, foi utilizada a escala Strength of Recommendation Taxonomy (SORT) da American Family Physician. $^{8}$

\section{RESULTADOS}

A pesquisa efetuada resultou na identificação de 181 artigos, tendo sido excluídos 174 por estarem duplicados, por já terem sido incluídos no sistema ou nas revisões sistemáticas ou por serem discordantes do objetivo e incluídos sete, sendo todos estes meta-análises (Figura 1). 


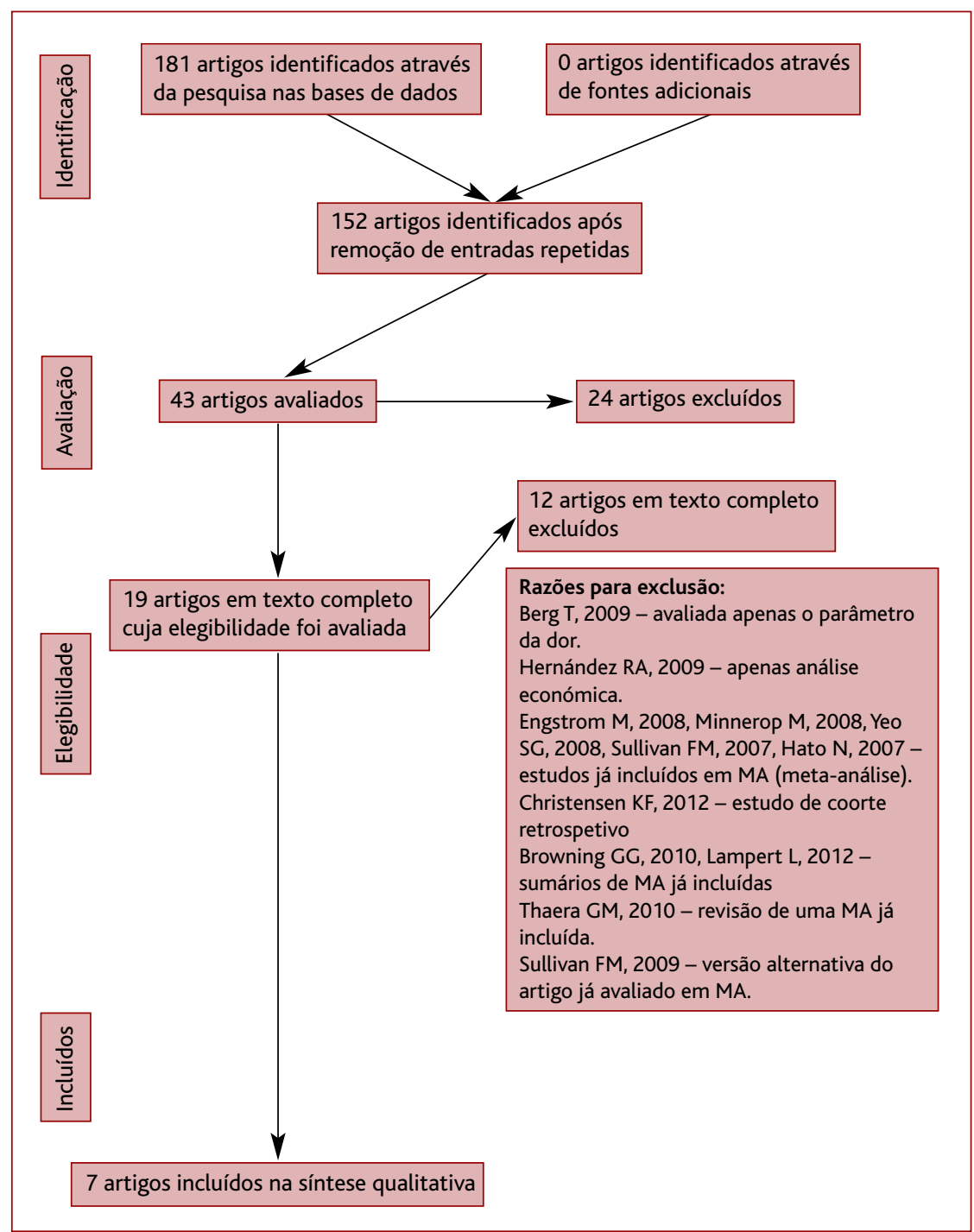

Figura 1. Resultados obtidos e artigos excluídos (baseado nas normas PRISMA, disponíveis em http://www.prisma-statement.org/2.1.4\%20-\%20PRISMA\%20Flow\%202009\%20Diagram. pdf).

\section{Associação de corticóides e antivíricos}

Na meta-análise de Almeida JR, 2009, cujo objetivo consistia na comparação da eficácia da associação de corticoides com antivíricos versus corticoides ou antivíricos isolados no tratamento da PFPI, foram incluídos 18 ensaios clínicos aleatorizados. ${ }^{3}$ Em três dos ensaios foi utilizada a associação de corticoides com antivíricos, em oito dos ensaios os corticoides foram utilizados de forma isolada, o mesmo acontecendo com os antivíricos em sete dos ensaios clínicos. Os outcomes pri- mários consistiam na recuperação da função motora do nervo facial, tendo em conta a severidade inicial, a modalidade do tratamento, a dose e o tempo de início de tratamento. Os outcomes secundários consistiam na disfunção sincinética e autonómica e nos efeitos adversos. Foram utilizadas as escalas House-Brackman, Facial Paralysis Recovery Index, Sunnybrook Scale, Yanagihara Score e Modified Adour Mechelse. Os resultados (Quadro I) mostram que os corticoides isolados permitiram uma recuperação satisfatória, com redução do risco de disfunção sincinética e autonómica e sem aumento do número de efeitos adversos. Os antivíricos isolados não foram associados a uma recuperação satisfatória. A associação das duas classes farmacológicas, embora permitisse uma maior taxa de recuperação comparativamente ao corticoide isolado, não teve significado estatístico.

A meta-análise de Goudakos JK, 2009, incluiu cinco ensaios clínicos aleatorizados, dos quais quatro puderam ser submetidos a meta-análise. ${ }^{5}$ Os outcomes primários consistiam na recuperação completa da função motora do nervo facial aos três, quatro, seis e nove meses (escala House-Brackmann). Os resultados (Quadro I) mostram uma taxa de recuperação motora semelhante entre os dois grupos aos três, quatro, seis e nove meses. A análise por sub-grupos não mostrou benefício no tratamento nos primeiros três dias após o início dos sintomas versus o tratamento três dias ou mais após o início dos sintomas. A adição do antivírico (aciclovir ou valaciclovir) ao tratamento com corticoide não produziu benefício adicional.

Na meta-análise de Quant EC, 2009, foram incluídos seis ensaios clínicos aleatorizados. ${ }^{9}$ No que diz respei- 
QUADRO I. Resultados obtidos nos diferentes estudos selecionados.

\begin{tabular}{|c|c|c|}
\hline Artigo & Resumo & NE \\
\hline \multirow{2}{*}{$\begin{array}{l}\text { Almeida } \\
\text { JR et al } \\
\text { (2009) }\end{array}$} & $\begin{array}{l}\text { Objetivo: Comparar CE+AV vs CE ou AV isolados ( } 2786 \text { doentes; seguimento } 6 \text { meses). } \\
\text { Intervenção: Prednisolona } 450 \text { mg (ou equivalente); Valaciclovir } 3000 \text { mg/d; Aciclovir } 4000 \text { mg/d. }\end{array}$ & \multirow[t]{2}{*}{1} \\
\hline & $\begin{array}{l}\text { Resultados: CE isolados: recuperação satisfatória, redução do risco de disfunção sincinésica e autonómica e } \\
\text { sem aumento de efeitos adversos. } \\
\text { AV isolados não foram associados com recuperação satisfatória. } \\
\text { AV+CE maior recuperação satisfatória (mas sem significado estatístico). } \\
\text { Poucos efeitos adversos (FA, úlcera gástrica), sem significado estatístico. }\end{array}$ & \\
\hline \multirow[t]{2}{*}{$\begin{array}{l}\text { Goudakos } \\
\text { JK et al. } \\
(2009)\end{array}$} & $\begin{array}{l}\text { Objetivo: Comparação da eficácia AV+CE vs CE isolados ( } 709 \text { doentes; seguimento } 3 \text { a } 9 \text { meses). } \\
\text { Intervenção: Prednisolona } 25 \text { mg, } 2 \text { id, } 10 \text { dias vs CE+aciclovir } 400 \text { mg, } 5 \text { id, } 10 \text { dias; prednisolona } 20 \text { mg, } \\
3 \text { id, } 10 \text { dias, vs CE+valaciclovir } 500 \text { mg, } 2 \text { id, } 5 \text { dias; prednisolona } 1 \mathrm{mg} / \mathrm{kg}, 10 \text { dias vs CE+aciclovir } 2000 \text { mg/d, } \\
10 \text { dias; prednisolona } 1 \mathrm{mg} / \mathrm{kg}, 12 \text { dias vs CE+aciclovir } 2400 \mathrm{mg} / \mathrm{d}, 10 \text { dias; deflazacort } 60 \mathrm{mg} / \mathrm{d}, 9 \text { dias } \\
\text { vs CE+valaciclovir } 1500 \mathrm{mg}, 1 \text { id, } 7 \text { dias. }\end{array}$ & \multirow[t]{2}{*}{1} \\
\hline & $\begin{array}{l}\text { Resultados: Taxa de recuperação motora igual entre os dois grupos aos 3, 4, } 6 \text { e } 9 \text { meses. } \\
\text { A adição do AV (aciclovir ou valaciclovir) ao tratamento com CE não produziu benefício adicional. } \\
\text { Efeitos adversos semelhantes entre os } 2 \text { grupos. }\end{array}$ & \\
\hline \multirow[t]{2}{*}{$\begin{array}{l}\text { Quant EC } \\
\text { et al. } \\
\text { (2009) }\end{array}$} & $\begin{array}{l}\text { Objetivo: Determinar benefício de CE+AV versus CE isolados (1145 doentes; seguimento mínimo de } 1 \text { mês) } \\
\text { Intervenção: Prednisolona } 1 \text { mg/kg, } 10 \text { dias vs CE+aciclovir } 2000 \text { mg/d, } 10 \text { dias; prednisolona } 60 \text { mg/d, } 10 \\
\text { dias vs CE+ valaciclovir } 1000 \text { mg/d, } 5 \text { dias; prednisolona } 50 \text { mg/d, } 10 \text { dias vs CE+aciclovir } 2000 \text { mg/d, } 10 \text { dias; } \\
\text { prednisolona } 60 \text { mg/d, } 10 \text { dias vs CE+valaciclovir } 3000 \text { mg/d, } 7 \text { dias; prednisolona } 1 \text { mg/kg, } 12 \text { dias vs } \\
\text { CE+famciclovir } 250 \text { mg/d, } 7 \text { dias; prednisolona } 1 \text { mg/kg, } 10 \text { dias vs CE+aciclovir } 2400 \text { mg/d, } 5 \text { dias. }\end{array}$ & \multirow[t]{2}{*}{1} \\
\hline & $\begin{array}{l}\text { Resultados: Não foi observado benefício da combinação AV + CE versus CE isolado. } \\
\text { A análise por sub-grupos (tempo até início do tratamento ou de seguimento, tipo de AV) manteve estas } \\
\text { conclusões. }\end{array}$ & \\
\hline \multirow{2}{*}{$\begin{array}{l}\text { Salinas } \\
\text { RA, et al. } \\
\text { (2010) }\end{array}$} & $\begin{array}{l}\text { Objetivo: Determinar eficácia e segurança da utilização de CE (1569 doentes; seguimento } 6 \text { meses ou até } \\
\text { recuperação total). }\end{array}$ & \multirow[t]{2}{*}{1} \\
\hline & $\begin{array}{l}\text { Resultados: Benefício significativo da utilização de CE na PFPI. } \\
\text { Redução significativa na sincinesia motora ao longo do seguimento naqueles que recebem CE. }\end{array}$ & \\
\hline \multirow[t]{2}{*}{$\begin{array}{l}\text { Lockhart } \\
\text { P et al. } \\
\text { (2010) }\end{array}$} & $\begin{array}{l}\text { Objetivo: Determinação da eficácia e segurança da utilização de AV (1987 utentes; seguimento: } 3 \text { a } 12 \\
\text { meses). } \\
\text { Intervenção: Comparados AV (aciclovir, valaciclovir, famciclovir) com placebo ou com CE ou a associação de } \\
\text { AV+C vs placebo. }\end{array}$ & \multirow[t]{2}{*}{1} \\
\hline & $\begin{array}{l}\text { Resultados: AV não foram mais eficazes do que placebo na recuperação completa. } \\
\text { É significativamente menos provável uma recuperação completa com AV do que com CE. Não há diferença } \\
\text { entre os diversos AV usados. } \\
\text { Efeitos adversos semelhantes ao placebo. }\end{array}$ & \\
\hline
\end{tabular}

to aos outcomesprimários, consistiam na proporção de doentes com, pelo menos, recuperação parcial no fim do intervalo de seguimento (definido como House-Brackmann $\geq 2$ ). Os resultados (Quadro I) mostram que não foi observado benefício da combinação de corticoide e antivírico versus corticoide isolado. A análise por sub-grupos (tempo até ao início do tratamento ou de seguimento, tipo de antivíricos) manteve as mesmas conclusões.

Na meta-análise de van der Veen EL, 2012, foram incluídos oito ensaios clínicos aleatorizados. ${ }^{11}$ Os outcomes primários consistiam na recuperação da função 


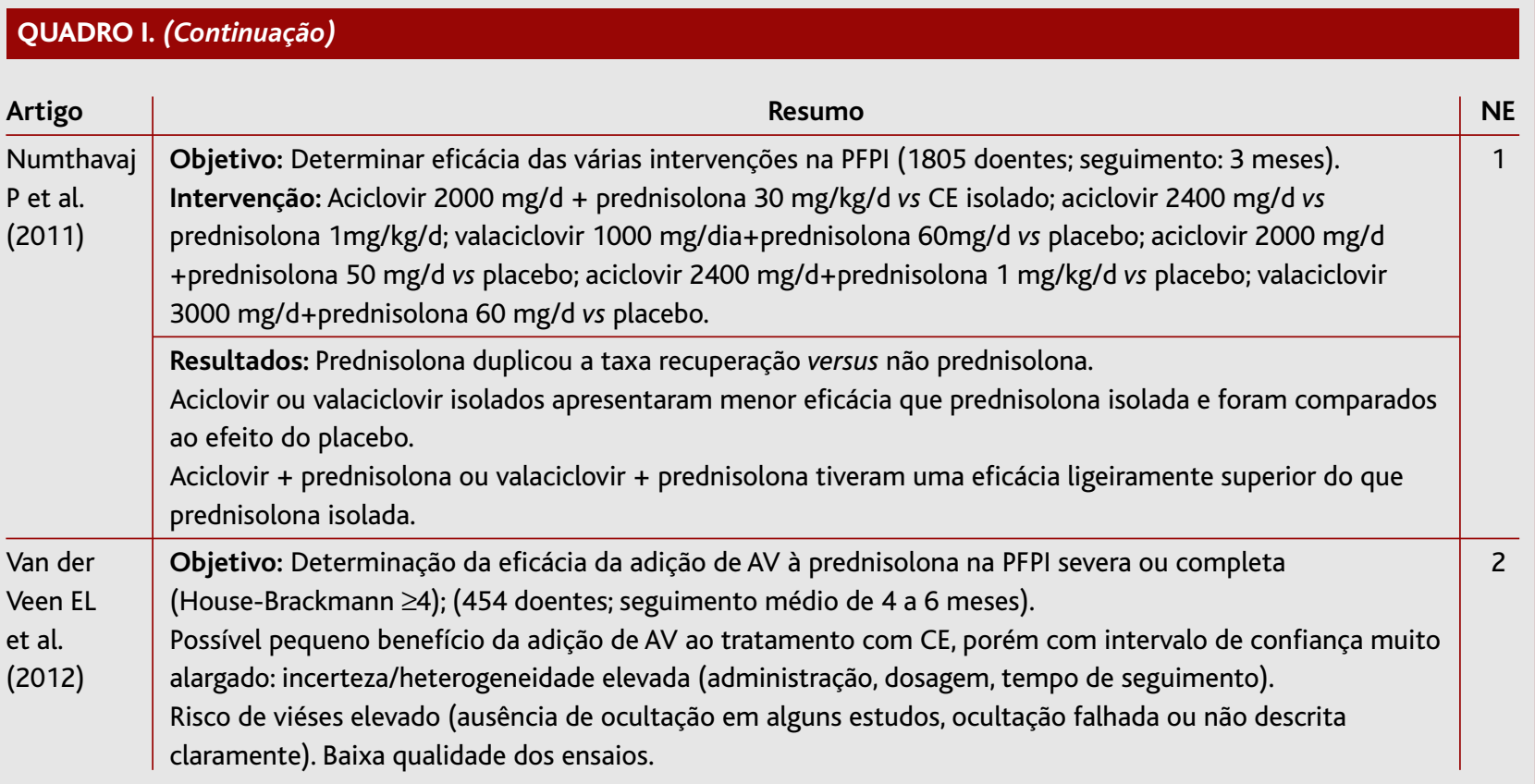

Legenda: CE - corticosteroide; AV - antivírico; FA - fibrilhação auricular; MA - meta-análise; NE - nível de evidência; id - número de vezes por dia; $\mathrm{mg} / \mathrm{d}$ - miligramas por dia; $\mathrm{mg} / \mathrm{kg} / \mathrm{d}$ - miligramas por quilograma de peso por dia; PFPI - paralisia facial periférica idiopática.

motora do nervo facial (escala House-Brackman). Os resultados (tabela I) mostram que existe um efeito benéfico, embora pequeno, na adição de antivírico ao tratamento com corticoide na paralisia severa, mas sem significado estatístico. Quanto aos efeitos secundários, foram encontrados entre 10 a 19\% com a utilização de corticoide isolado, e 13 a $20 \%$ com a associação de corticoide e antivírico e consistiam geralmente em efeitos gastrointestinais ou tonturas, sem necessidade de tratamento subsequente.

Na meta-análise de Numthavaj P, 2011, foram incluídos seis ensaios clínicos aleatorizados. ${ }^{2}$ Cinco dos ensaios compararam a utilização da combinação de antivírico e prednisolona com prednisolona ou antivírico isolados ou placebo, enquanto o outro ensaio comparou a utilização de antivírico com prednisolona. Em quatro dos ensaios o antivírico utilizado foi o aciclovir, enquanto noutros dois estudos foi utilizado o valaciclovir. Os outcomes primários consistiam na recuperação da função motora do nervo facial, definida de acordo com as seguintes pontuações nas escalas de avalia- ção: House-Brackmann $\leq 2$, Facial Palsy Recovery Index $\geq 8$, $>36$ pontos no Yanagihara Score ou 100 na Sunnybrook Scale. Os resultados (tabela I) mostraram que a prednisolona duplicou a taxa de recuperação comparativamente à sua não utilização. $\mathrm{O}$ aciclovir ou valaciclovir isolados apresentaram uma menor eficácia que a prednisolona isolada e tiveram efeitos semelhantes ao placebo. A combinação do aciclovir ou valaciclovir com prednisolona teve uma eficácia ligeiramente superior à prednisolona isolada, embora esta diferença não atinja significado estatístico.

\section{Corticóides isolados}

Na meta-análise de Salinas RA, 2010 (Cochrane), foram incluídos oito ensaios clínicos aleatorizados..$^{10} \mathrm{Em}$ todos os ensaios foi comparada a utilização de corticoide com placebo, sendo maioritariamente utilizada a prednisolona. No que diz respeito aos outcomes primários, consistiam na recuperação incompleta da função motora facial após seis meses ou mais, no desenvolvimento de sincinesias motoras ou disfunção auto- 
nómica (espasmo hemifacial) e nos efeitos adversos. Foi utilizada a escala de House-Brackmann. Os resultados (tabela I) demonstram um benefício significativo da utilização de corticoides na PFPI, assim como uma redução significativa na sincinesia motora naqueles que receberam corticoides versus placebo.

\section{Antivíricos isolados}

A meta-análise de Lockhart P, 2010 (Cochrane), incluiu sete ensaios clínicos aleatorizados. ${ }^{4}$ Os outcomes primários consistiam na recuperação da função motora do nervo facial no fim do estudo (escala de House-Brackmann), na disfunção sincinética e autonómica e nos efeitos adversos. Os resultados (Quadro I) mostram que os antivíricos não foram mais eficazes do que o placebo na recuperação completa da função motora do nervo facial. Foi significativamente menos provável uma recuperação completa com os antivíricos do que com os corticoides. Não houve diferença significativa entre os diversos antivíricos utilizados e os efeitos adversos foram semelhantes entre as diversas combinações terapêuticas em estudo.

\section{CONCLUSÕES}

Esta revisão apresenta algumas limitações: há que considerar a elevada heterogeneidade e limitações metodológicas dos ensaios aleatorizados nos quais as meta-análises se baseiam, nomeadamente no que concerne à definição de outcomes, tempo de seguimento, tempo até à melhoria clínica, medicação e doses usadas, estratificação da gravidade, análises post-hoc de subgrupos, bem como a garantia da aleatorização e dupla ocultação adequadas. A limitação linguística pode eventualmente levar à não inclusão de estudos potencialmente relevantes em outras línguas.

Deve salientar-se, com base na evidência disponível, que existe um benefício significativo, consistente e orientado para o doente, no uso de corticoides, mas não na utilização isolada (ou em combinação) de antivíricos, os quais comportam custos adicionais (sem um benefício comprovado). Adicionalmente deve-se ter em conta o facto de que muitos dos doentes melhoram espontaneamente, de forma completa. Por fim, as comorbilidades do doente (com o seu possível impacto nas interações e, portanto, efeitos secundários), assim como a probabilidade do doente ter uma adesão terapêutica adequada perante um esquema de combinação (que será mais complexo e dispendioso) não devem ser esquecidas.

Em suma, não existe evidência de um benefício clínico dos antivíricos, isoladamente ou em associação com os corticoides, no tratamento da Paralisia de Bell (Força de Recomendação B). Os corticoides continuam a ser o tratamento farmacológico com a melhor evidência clínica, relevante para o doente, disponível (Força de Recomendação A). Não foram encontrados efeitos adversos significativos com a utilização de corticoides, antivíricos ou a sua combinação.

\section{REFERÊNCIAS BILIOGRÁFICAS}

1. Tiemstra JD, Khatkhate N. Bell's palsy: diagnosis and management. Am Fam Physician 2007 Oct 1; 76 (7): 997-1002.

2. Numthavaj P, Thakkinstian A, Dejthevaporn C,Attia J. Corticosteroid and antiviral therapy for Bell's palsy: a network meta-analysis. BMC Neurol 2011 Jan 5; 11: 1.

3. de Almeida JR, Al Khabori M, Guyatt GH, Witterick IJ, Lin VY, Nedzelski JM, Chen JM, et al. Combined corticosteroid and antiviral treatment for Bell palsy: a systematic review and meta-analysis. JAMA 2009 Sep 2; 302 (9): 985-93.

4. Lockhart P, Daly F, Pitkethly M, Comerford N, Sullivan F. Antiviral treatment for Bell's palsy (idiopathic facial paralysis). Cochrane Database Syst Rev 2010 Oct 7; (4): CD001869.

5. Goudakos JK, Markou KD. Corticosteroids vs corticosteroids plus antiviral agents in the treatment of Bell palsy: a systematic review and meta-analysis. Arch Otolaryngol Head Neck Surg. 2009 Jun; 135 (6): 558-64.

6. Gilden DH, Tyler KL. Bell's palsy - is glucocorticoid treatment enough? N Engl J Med 2007 Oct 18; 357 (16): 1653-5.

7. Gilden DH. Clinical practice: Bell's Palsy. N Engl J Med 2004 Sep 23; 351 (13): 1323-31.

8. Ebell MH, Siwek J, Weiss BD, Woolf SH, Susman J, Ewingman B, et al. Strenght of recommendation taxonomy (SORT): a patient-centered approach to grading evidence in the medical literature. Am Fam Phys 2004 Feb 1; 69 (3): 548-56.

9. Quant EC, Jeste SS, Muni RH, Cape AV, Bhussar MK, Peleg AY. The benefits of steroids versus steroids plus antivirals for treatment of Bell's palsy: a meta-analysis. BMJ 2009 Sep 7; 339: b3354.

10. Salinas RA, Alvarez G, Daly F, Ferreira J. Corticosteroids for Bell's palsy (idiopathic facial paralysis). Cochrane Database Syst Rev 2010; (3): CD001942.

11. van der Veen EL, Rovers MM, de Ru JA, van der Heijden GJ. A small effect of adding antiviral agents in treating patients with severe Bell palsy. Otolaryngol Head Neck Surg 2012 Mar; 146 (3): 353-7.

\section{CONFLITOS DE INTERESSE}

Os autores declaram não ter conflito de interesses. 
ENDEREÇO PARA CORRESPONDÊNCIA

Ana Isabel Silva

Estrada da Granja, 114

Valbom

4420-465 GONDOMAR

E-mail: anisabelfs@gmail.com
Recebido em 22/08/2012

Aceite para publicação em 08/09/2013

Artigo escrito ao abrigo do novo acordo ortográfico.

\section{ABSTRACT}

\section{PHARMACOLOGICAL TREATMENT OF IDIOPATHIC FACIAL NERVE PARALYSIS: WHAT IS THE EVIDENCE?}

Objective: The objective of this study was to review the evidence for the most effective pharmacological treatment for idiopathic peripheral facial paralysis using corticosteroids and antiviral agents.

Data sources: PubMed, evidence based medicine websites, Portuguese Medical Journal Index and references of selected articles were searched.

Methodology: An electronic database search was conducted for clinical guidelines, systematic reviews, meta-analysis (MA) and randomized controlled clinical trials, published between 01/2005 and 04/2012, in English and Portuguese, using the MeSH terms: Glucocorticoids; Antiviral Agents; Bell Palsy; Facial Paralysis. The Strength of Recommendation Taxonomy (SORT) of the American Family Physician was used for the assessment of levels of evidence (LE) and the strength of recommendations.

Results: Of the 181 articles found, seven MA were included. Three MA found significant clinical benefit from corticosteroid therapy compared to placebo treatment (LE 1). The use of antiviral drugs was no better than placebo in two studies (LE 2). The combination of corticosteroids and antiviral drugs was found to be similar to isolated corticosteroid therapy in two studies. In four studies, increased benefit from combination therapy was found compared to corticosteroid treatment alone, though this difference was not statistically significant. Benefit from the combination treatment was possibly more likely in cases of severe paralysis (LE 2).

Conclusions: Evidence is strongest for the benefits of corticosteroids in the treatment of idiopathic facial nerve paralysis (Recommendation A). There is no evidence of benefit for antiviral drugs either alone or in combination with corticosteroids in the treatment of Bell's palsy (Recommendation B). In patients with severe paralysis, there may be a small benefit in adding an antiviral drug to steroid therapy (Recommendation B). Numerous methodological limitations in the reported studies as well as the heterogeneity between studies were noted.

Keywords: Idiopathic Peripheral Facial Palsy; Bell's Palsy; Corticosteroids; Antivirals. 